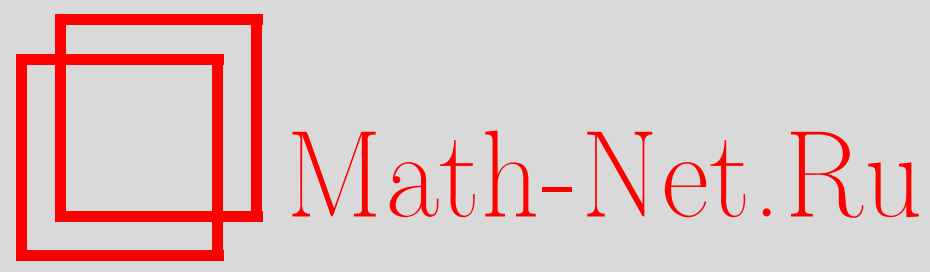

А. Н. Бахвалов, О $\lambda$-расходимости ряда Фурье непрерывной функции многих переменных, Матем. заметки, 2002, том 72, выпуск 4, 490-501

DOI: https://doi.org/10.4213/mzm438

Использование Общероссийского математического портала Math-Net.Ru подразумевает, что вы прочитали и согласны с пользовательским соглашением http://www.mathnet.ru/rus/agreement

Параметры загрузки:

IP: 52.6 .47 .48

26 апреля 2023 г., $15: 36: 58$

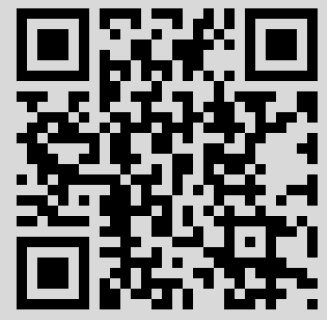




\section{О $\lambda$-РАСХОДИМОСТИ РЯДА ФУРЬЕ НЕПРЕРЫВНОЙ ФУНКЦИИ МНОГИХ ПЕРЕМЕННЫХ}

\section{А. Н. Бахвалов}

В статье рассматривается поведение прямоугольных частичных сумм рядов Фурье непрерывных функций многих переменных по тригонометрической системе. Ряд Фурье называется $\lambda$-сходящимся, если существует предел прямоугольных частичных сумм по индексам $\vec{M}=\left(M_{1}, \ldots, M_{n}\right)$, для которых $1 / \lambda \leqslant M_{j} / M_{k} \leqslant \lambda$ для всех $j$ и $k$. В пространстве произвольной четной размерности $2 m$ построен пример непрерывной функции с оценкой на модуль непрерывности $\omega(F, \delta)=\underset{\delta \rightarrow+0}{O}\left(\ln ^{-m}(1 / \delta)\right)$, ряд Фурье которой $\lambda$-расходится всюду для любого $\lambda>1$.

Библиография: 9 названий.

1. Введение. Пусть $f(\vec{x}) 2 \pi$-периодична по каждому аргументу и интегрируема по Лебегу на $\mathbb{T}^{n}, \mathbb{T}=[0 ; 2 \pi)$. Ее яядм ярье по тригонометрической системе назьвается ряд

$$
\sum_{m_{1}, \ldots, m_{n}=-\infty}^{+\infty} c_{m} e^{i\langle\vec{m}, \vec{x}\rangle}
$$

где

$$
c_{\vec{m}}=c_{m_{1}, \ldots, m_{n}}=\frac{1}{(2 \pi)^{n}} \iint_{\mathbb{T}^{n}} f(\vec{x}) e^{-i\langle\vec{m}, \vec{x}\rangle} d x_{1} \cdots d x_{n}
$$

- коэффициенты Фурье функции $f$.

Прямоугольной частичной суммой ряда Фурье назьвается

$$
S_{\vec{M}}(f, \vec{x})=S_{M_{1}, \ldots, M_{n}}(f, \vec{x})=\sum_{m_{1}=-M_{1}}^{M_{1}} \ldots \sum_{m_{n}=-M_{n}}^{M_{n}} c_{\vec{m}} e^{i\langle\vec{m}, \vec{x}\rangle} .
$$

Ряд Фурье функции $f$ назьвается $\lambda$-сходящимся (по прямоугольникам) в точке $\vec{x}$, где $\lambda \geqslant 1$, если существует

$$
\min _{\min _{j} \rightarrow+\infty} S_{\vec{M}}(f, \vec{x})
$$

по всем номерам $\vec{M}$ таким, что $1 / \lambda \leqslant M_{j} / M_{k} \leqslant \lambda$ для любых $j$ и $k$.

Работа выполнена при финансовой поддержке Российского фонда фундаментальных исследований, грант № 00-01-00042, и программы “Ведушие научњые школы", грант № 00-15-96143. 
1-сходимость обычно называется сходимостью по квадратам (по кубам), а сходимость без ограничений на отношения компонент - сходимостью по прямоугольникам (по Прингсхейму).

Модулем непрерывности $2 \pi$-периодической по каждому аргументу функции $f \in$ $C\left(\overline{\mathbb{T}^{n}}\right)$ назьвается функция от $\delta>0$, определяемая формулой

$$
\omega(f, \delta)=\sup _{\vec{x} \in \mathbb{R}^{n}} \sup _{\vec{h} \in \mathbb{R}^{n}:\|\vec{h}\| \leqslant \delta}|f(\vec{x}+\vec{h})-f(\vec{x})| .
$$

Как следует из результатов Феффермана [1], для любой непрерьвной функции многих переменных ее ряд Фурье сходится по кубам почти всюду.

В другой работе Феффермана [2] был впервые построен пример непрерьвной функции двух переменных, ряд Фурье которой расходится по Прингсхейму всюду. Основываясь на методе Феффермана, Бахбух и Никишин [3] построили пример функции двух переменных с оценкой на модуль непрерьвности

$$
\omega(f, \delta)=\underset{\delta \rightarrow+0}{O}\left(\ln ^{-1} \frac{1}{\delta}\right),
$$

ряд Фурье которой расходится по Прингсхейму на $[0.2 ; 2 \pi-0.2]^{2}$.

С другой стороны, Осколков [4] и Жижиашвили [5] получили достаточные условия сходимости ряда Фурье почти всюду по Прингсхейму в терминах модулей непрерывности.

Как отметил Дьяченко [6], незначительная модификация рассуждений Бахбуха и Никишина позволяет показать, что для любого натурального $m$ существует непрерьвная $2 \pi$-периодическая по каждому переменному функция $2 m$ переменных $f(\vec{x})$ такая, что $\omega(f, \delta)=O\left((\ln 1 / \delta)^{-m}\right)$ при $\delta \rightarrow+0$ и ее ряд $\Phi$ урье расходится по Прингсхейму почти всюду.

Автором в работе [7] было установлено, что эти результаты можно усилить следующим образом.

Теорема А. Для любого $\lambda>1$ существует функиия $H(\vec{x}) \in C\left(\overline{\mathbb{T}^{2 m}}\right)$ такая, что ее ряд Фурье $\lambda$-расходится всюду, а ее модуль непрерывности удовлетворяет условию

$$
\omega(H, \delta)=\underset{\delta \rightarrow+0}{O}\left(\ln ^{-m}\left(\frac{1}{\delta}\right)\right) .
$$

Целью настоящей работы является построение непрерывной функции многих переменных, ряд Фурье которой $\lambda$-расходится всюду для любого выбранного $\lambda>1$ (в отличие от теоремы А, где функция строилась для фиксированного $\lambda$ ). Получен следующий основной результат.

ТЕорема 1. Для любого натурального $m$ существует функиия $F(\vec{x}) \in C\left(\overline{\mathbb{T}^{2 m}}\right)$ такая, что ее ряд Фурье $\lambda$-расходится всюду для любого $\lambda>1$, а ее модуль непрерывности удовлетворяет условию

$$
\omega(F, \delta)=\underset{\delta \rightarrow+0}{O}\left(\ln ^{-m}\left(\frac{1}{\delta}\right)\right) .
$$


2. Основные элементы построения. В этом пункте мы рассматриваем рядвспомогательных утверждений. Некоторые из них являются незначительными видоизменениями результатов из [3] и [7] и поэтому приводятся без доказательств.

Пусть $p$ - натуральное число. Положим $\lambda_{p}=(p+1) / p$ и $D_{p}=2 p+1 \geqslant 3$; тогда $D_{p}=\left(\lambda_{p}+1\right) /\left(\lambda_{p}-1\right)$. Положим $A_{p, 1}=\pi-\pi / D_{p}$ и $A_{p, 2}=2 \pi-A_{p, 1}$; отметим, что $A_{p, 2} / A_{p, 1}=\lambda_{p}$. Действительно,

$$
\frac{A_{p, 2}}{A_{p, 1}}=\frac{1+1 / D_{p}}{1-1 / D_{p}}=\frac{D_{p}+1}{D_{p}-1}=\frac{2 p+2}{2 p}
$$

Обозначим также $\Delta_{p}=2 \pi / D_{p}=A_{p, 2}-A_{p, 1}$.

Введем обозначения для промежутков:

$$
I_{p, 1}=\left[A_{p, 1}-\Delta_{p}, A_{p, 1}\right), \quad I_{p, 2}=\left(A_{p, 1}, A_{p, 2}\right), \quad I_{p, 3}=\left(A_{p, 2}, A_{p, 2}+\Delta_{p}\right]
$$

и для квадратов:

$$
Q_{j, k, p}=I_{p, j} \times I_{p, k}, \quad j, k=1,2,3 .
$$

Зададим функцию $g_{\nu, p}(x, y)$ на квадратах $Q_{j, k, p}$ формулой

$$
g_{\nu, p}(x, y)= \begin{cases}e^{i \nu x y}, & (x, y) \in Q_{2,2, p} \\ \left(\Delta_{p}\right)^{-1}\left(x-A_{p, 1}+\Delta_{p}\right) e^{i \nu A_{p, 1} y}, & (x, y) \in Q_{1,2, p} \\ \left(\Delta_{p}\right)^{-1}\left(y-A_{p, 1}+\Delta_{p}\right) e^{i \nu A_{p, 1} x}, & (x, y) \in Q_{2,1, p} \\ \left(\Delta_{p}\right)^{-1}\left(A_{p, 2}+\Delta_{p}-x\right) e^{i \nu A_{p, 2} y}, & (x, y) \in Q_{3,2, p} \\ \left(\Delta_{p}\right)^{-1}\left(A_{p, 2}+\Delta_{p}-y\right) e^{i \nu A_{p, 2} x}, & (x, y) \in Q_{2,3, p} \\ \left(\Delta_{p}\right)^{-2}\left(x-A_{p, 1}+\Delta_{p}\right)\left(y-A_{p, 1}+\Delta_{p}\right) e^{i \nu A_{p, 1}^{2},} & (x, y) \in Q_{1,1, p} \\ \left(\Delta_{p}\right)^{-2}\left(A_{p, 2}+\Delta_{p}-x\right)\left(y-A_{p, 1}+\Delta_{p}\right) e^{i \nu A_{p, 1} A_{p, 2}}, & (x, y) \in Q_{3,1, p} \\ \left(\Delta_{p}\right)^{-2}\left(x-A_{p, 1}+\Delta_{p}\right)\left(A_{p, 2}+\Delta_{p}-y\right) e^{i \nu A_{p, 1} A_{p, 2},}, & (x, y) \in Q_{1,3, p} \\ \left(\Delta_{p}\right)^{-2}\left(A_{p, 2}+\Delta_{p}-x\right)\left(A_{p, 2}+\Delta_{p}-y\right) e^{i \nu A_{p, 2}^{2},} & (x, y) \in Q_{3,3, p}\end{cases}
$$

На граничных точках квадратов доопределим ее по непрерьвности. На остальной части $\mathbb{T}^{2}$ доопределим функцию нулем. Продолжим ее $2 \pi$-периодически на всю плоскость.

Для $\nu, p \in \mathbb{N}$ и $j, k=1,2,3$ обозначим

$$
g_{\nu, p}^{j, k}(x, y)=g_{\nu, p}(x, y) \chi_{Q_{j, k, p}}(x, y)
$$

Полученные функции продолжим $2 \pi$-периодически на всю плоскость. Отметим, что после продолжения $g_{\nu, p}$ непрерьвна на $\mathbb{R}^{2}$.

ЛЕмма 1. Для вещественных $а$ и $b$ и натурального $p$ при $|a|>2|b|>2$ или $|b|>2|a|>2$ для любого $x \in \mathbb{T}$ выполняется оченка

$$
\left|\int_{A_{p, 1}}^{A_{p, 2}} \frac{\sin a(t-x)}{t-x} e^{i b t} d t\right|<15 .
$$


ДоКАЗАТЕЛЬСТВо аналогично доказательству леммы 4 из [7].

Положим для функции $f \in L\left(\mathbb{T}^{2}\right)$

$$
\widetilde{S}_{M, N}(f, x, y)=\frac{1}{\pi^{2}} \iint_{\mathbb{T}^{2}} \frac{\sin M(s-x)}{s-x} \frac{\sin N(t-y)}{t-y} f(s, t) d s d t .
$$

Лемма 2. Существует абсолютная постоянная $C_{1}$ такая, что при $\nu>100$ для любой точки $(x, y) \in \mathbb{T}^{2}$ и номеров $M$ и $N$ таких, что выполняется пара неравенств

$$
M^{2} \leqslant 2 \nu, \quad N^{2} \leqslant 2 \nu,
$$

имеет место оценка

$$
\left|S_{M, N}\left(g_{\nu, p}^{2,2},(x, y)\right)\right|<C_{1}+30(\ln \min \{M, N\}+3) .
$$

ДокАЗАТЕЛьСтво. Покажем сначала, что при условиях леммы имеет место неравенство

$$
\left|S_{M, N}\left(g_{\nu, p}^{2,2},(x, y)\right)-\widetilde{S}_{M, N}\left(g_{\nu, p}^{2,2},(x, y)\right)\right|<C_{1},
$$

где $\widetilde{S}_{M, N}\left(g_{\nu, p}^{2,2},(x, y)\right)$ определено равенством (2).

Введем обозначение

$$
\psi(a, s, q)=\frac{\sin ((a+1 / 2)(s-q))}{2 \sin ((s-q) / 2)}-\frac{\sin (a(s-q))}{s-q} .
$$

Заметим, что

$$
\begin{aligned}
\psi(a, s, q)= & \frac{\sin a(s-q)}{2 \sin ((s-q) / 2)}\left(\cos \frac{s-q}{2}-1\right)+\frac{1}{2} \cos a(s-q) \\
& +\sin a(s-q)\left(\frac{1}{2 \sin ((s-q) / 2)}-\frac{1}{s-q}\right)=r_{1}+r_{2}+r_{3} .
\end{aligned}
$$

Здесь $\left|r_{2}\right| \leqslant 1 / 2$. Поскольку при $A_{p, 1} \leqslant s \leqslant A_{p, 2}$ и $0 \leqslant q \leqslant 2 \pi$ выполнено $|q-s| \leqslant 2 \pi-$ $A_{p, 1} \leqslant 4 \pi / 3$, то

$$
\left|r_{3}\right| \leqslant\left|\left(\frac{1}{2 \sin ((s-q) / 2)}-\frac{1}{s-q}\right)\right| \leqslant \rho_{0}
$$

и

$$
\begin{aligned}
\left|r_{1}\right| & =\left|\frac{\sin a(s-q)}{4 \sin ((s-q) / 4) \cos ((s-q) / 4)} 2 \sin ^{2} \frac{s-q}{4}\right| \leqslant\left|\frac{1}{2 \cos ((s-q) / 4)}\right| \\
& \leqslant \frac{1}{2 \cos \left(\left(2 \pi-A_{p, 1}\right) / 4\right)} \leqslant 1 .
\end{aligned}
$$

Складьвая, получаем

$$
|\psi(a, s, q)| \leqslant \rho_{0}+1+\frac{1}{2}=\psi_{0}
$$


Теперь имеем

$$
\begin{aligned}
& S_{M, N}\left(g_{\nu, p}^{2,2},(x, y)\right)-\widetilde{S}_{M, N}\left(g_{\nu, p}^{2,2},(x, y)\right) \\
& =\iint_{Q_{2,2, p}} \frac{\sin (M+1 / 2)(s-x)}{2 \sin ((s-x) / 2)} \frac{\sin (N+1 / 2)(t-y)}{2 \sin ((t-y) / 2)} e^{i \nu s t} d s d t \\
& \quad-\iint_{Q_{2,2, p}} \frac{\sin M(s-x)}{s-x} \frac{\sin N(t-y)}{t-y} e^{i \nu s t} d s d t \\
& =\int_{A_{p, 1}}^{A_{p, 2}} \psi(N, t, y)\left(\int_{A_{p, 1}}^{A_{p, 2}} \frac{\sin M(s-x)}{s-x} e^{i \nu s t} d s\right) d t \\
& \quad+\int_{A_{p, 1}}^{A_{p, 2}} \psi(M, s, x)\left(\int_{A_{p, 1}}^{A_{p, 2}} \frac{\sin N(t-y)}{t-y} e^{i \nu s t} d t\right) d s \\
& \quad+\iint_{Q_{2,2, p}} e^{i \nu s t} \psi(M, s, x) \psi(N, t, y) d t d s \\
& =J_{1}+J_{2}+J_{3},
\end{aligned}
$$

где функшия $\psi(a, r, q)$ определена формулой (5) и равномерно ограничена при всех $a \in \mathbb{R}$, $A_{p, 1} \leqslant r \leqslant A_{p, 2}$ и $0 \leqslant q \leqslant 2 \pi$ величиной $\psi_{0}$ в силу (6). Отсюда и из леммы 1 , пользуясь тем, что при $s \in\left[A_{p, 1}, A_{p, 2}\right]$ в силу (3) справедливо неравенство

получаем оценки

$$
\frac{\nu s}{M} \geqslant \frac{2 \pi}{3} \sqrt{\frac{\nu}{2}}>2
$$

$$
\left|J_{1}\right|<\left(A_{p, 2}-A_{p, 1}\right) 15 \psi_{0}, \quad\left|J_{2}\right|<\left(A_{p, 2}-A_{p, 1}\right) 15 \psi_{0}, \quad\left|J_{3}\right|<\left(A_{p, 2}-A_{p, 1}\right)^{2} \psi_{0}^{2} .
$$

Теперь можно положить

$$
C_{1}=\frac{1}{\pi^{2}}\left(\left(A_{1,2}-A_{1,1}\right) 30 \psi_{0}+\left(A_{1,2}-A_{1,1}\right)^{2} \psi_{0}^{2}\right) .
$$

Тем самьм, (4) установлено.

Пусть теперь для определенности $M \geqslant N$; тогда

$$
\widetilde{S}_{M, N}\left(g_{\nu, p}^{2,2}, x, y\right)=\int_{A_{p, 1}}^{A_{p, 2}} \frac{\sin N(t-y)}{t-y}\left(\int_{A_{p, 1}}^{A_{p, 2}} e^{i \nu s t} \frac{\sin M(s-x)}{s-x} d s\right) d t .
$$

Заметим, что в силу условий (3) вьполнено неравенство $\nu t>2 \pi \nu / 3>2 M$. Применяя к внутреннему интегралу лемму 1 , получаем

$$
\left|\widetilde{S}_{M, N}\left(g_{\nu, p}^{2,2}, x, y\right)\right| \leqslant 15 \int_{A_{p, 1}}^{A_{p, 2}}\left|\frac{\sin N(t-y)}{t-y}\right| d t .
$$

Поскольку при $y \in \mathbb{T}$ вьполнено $2 \pi / 3 \leqslant\left|A_{p, k}-y\right| \leqslant 4 \pi / 3$, где $k=1,2$, то

$$
\begin{aligned}
\int_{A_{p, 1}}^{A_{p, 2}}\left|\frac{\sin N(t-y)}{t-y}\right| d t & \leqslant \int_{\left[A_{p, 1}, A_{p, 2}\right] \backslash[y-1 / N, y+1 / N]}\left|\frac{1}{t-y}\right| d t+\int_{y-1 / N}^{y+1 / N} N d t \\
& <2 \ln N+6 .
\end{aligned}
$$

Объединяя оценки (4), (7) и (8), получаем, что

$$
\left|S_{M, N}\left(g_{\nu, p}^{2,2},(x, y)\right)\right|<C_{1}+30(\ln \min \{M, N\}+3) .
$$

Лемма доказана. 
Лемма 3. Существует абсолютная постоянная $C_{2}$ такая, что при $\nu>100$ для любой точки $(x, y) \in \mathbb{T}^{2}$ и номеров $M$ и $N$ таких, что выполняется пара неравенств

$$
M^{2} \leqslant 2 \nu, \quad N^{2} \leqslant 2 \nu,
$$

имеет место оценка

$$
S_{M, N}\left(g_{\nu, p},(x, y)\right)<C_{2}(1+\ln \min \{M, N\})
$$

ДокАЗАТЕЛЬСТВо. В силу леммы 2 нам достаточно оценить частичные суммы $S_{M, N}\left(g_{\nu, p}^{j, k},(x, y)\right)$ при $j \neq 2$ или $k \neq 2$.

Рассмотрим случаи $j=k=1$ и $j=1, k=2$ (остальные случаи аналогичны).

В первом случае имеем

$$
\begin{aligned}
S_{M, N}\left(g_{\nu, p}^{1,1},(x, y)\right)= & e^{i \nu\left(A_{p, 1}\right)^{2}} \frac{1}{\pi} \int_{A_{p, 1}-\Delta_{p}}^{A_{p, 1}} \frac{s-A_{p, 1}+\Delta_{p}}{\Delta_{p}} D_{M}(s-x) d s \\
& \times \frac{1}{\pi} \int_{A_{p, 1}-\Delta_{p}}^{A_{p, 1}} \frac{t-A_{p, 1}+\Delta_{p}}{\Delta_{p}} D_{N}(t-y) d s .
\end{aligned}
$$

Таким образом, достаточно показать, что частичные суммы рядов Фурье функций

$$
\frac{x-A_{p, 1}+\Delta_{p}}{\Delta_{p}} \chi_{\left[A_{p, 1}-\Delta_{p}, A_{p, 1}\right)}(x)
$$

равномерно ограничены. Заметим, что эти функции (будучи продолженньми $2 \pi$-периодически) являются функциями ограниченной вариации на $\mathbb{T}$ и непрерывны всюду на $\mathbb{T}$, кроме точки $A_{p, 1}$, которая является точкой разрьва первого рода. При этом вариация функции на периоде равна 2 для любого $p$. Отсюда следует (см., например, [8]), что такие частичные суммы равномерно по $x$ и $p$ ограничены.

Во втором случае имеем

$$
\begin{aligned}
S_{M, N}\left(g_{\nu, p}^{1,2},(x, y)\right)= & \frac{1}{\pi} \int_{A_{p, 1}-\Delta_{p}}^{A_{p, 1}} \frac{s-A_{p, 1}+\Delta_{p}}{\Delta_{p}} D_{M}(s-x) d s \\
& \times \frac{1}{\pi} \int_{A_{p, 1}}^{A_{p, 2}} e^{i \nu A_{p, 1} t} D_{N}(t-y) d t .
\end{aligned}
$$

Первьй интеграл равномерно ограничен по указанным при разборе предыдущего случая соображениям, а второй - в силу леммы 1 и того, что (см. (5), (6))

$$
\left|\int_{A_{p, 1}}^{A_{p, 2}} e^{i \nu A_{p, 1} t} D_{N}(t-y) d t-\int_{A_{p, 1}}^{A_{p, 2}} e^{i \nu A_{p, 1} t} \frac{\sin N(t-y)}{t-y} d t\right| \leqslant \frac{2 \pi}{3} \psi_{0} .
$$

Лемма доказана.

Лемма 4. Пусть $0<\varepsilon<\Delta_{p} / 2 ;$ тогда существует $\nu_{0}=\nu_{0}(\varepsilon, p)$ такое, что для любой точки $(x, y) \in\left[A_{p, 1}+\varepsilon ; A_{p, 2}-\varepsilon\right]^{2}$ выполнено

$$
\forall \nu>\nu_{0} \quad\left|S_{M, N}\left(g_{\nu, p},(x, y)\right)\right| \geqslant \ln \nu \quad \partial \Omega_{\Omega} \quad M=[\nu y], \quad N=[\nu x] .
$$


ДоКАЗАТЕЛЬСТВо леммы аналогично доказательству леммы 8 из [7].

Лемма 5. Пусть $0<\varepsilon<\Delta_{p} / 2$. Тогда существует $C_{3}(\varepsilon, p)$ такая, что для любых натуральных $M$ и $N$ при любом $\nu>100$ и для любой точки $(x, y) \notin Q_{2,2, p}$ такой, что выполнены условия

$$
\left|x-A_{p, j}\right|>\varepsilon, \quad\left|y-A_{p, j}\right|>\varepsilon, \quad j=1,2,
$$

справедлива оченка

$$
\left|S_{M, N}\left(g_{\nu, p}(x, y)\right)\right| \leqslant C_{3}(\varepsilon, p) .
$$

Кроме того, существует $C_{4}(\varepsilon, p)$ такая, ито если $(x, y) \in\left[A_{p, 1}+\varepsilon ; A_{p, 2}-\varepsilon\right]^{2}$, то для любых натуральных $M$ и $N$ при любом $\nu>100$ выполнено

$$
\left|S_{M, N}\left(g_{\nu, p}(x, y)\right)\right| \leqslant C_{4}(\varepsilon, p) \ln \nu
$$

ДоКАЗАТЕЛЬСТво леммы аналогично доказательству леммы 9 из [7].

Перейдем теперь к рассмотрению функций $2 m$ переменных.

Положим

$$
\bar{g}_{\nu, p}(\vec{x})=\prod_{k=1}^{m} g_{\nu, p}\left(x_{2 k-1}, x_{2 k}\right) .
$$

Определим функцию $h_{\nu, p}(x, y)$ формулой

$$
h_{\nu, p}(\vec{x})=\sum_{j_{1}=-p}^{p} \ldots \sum_{j_{2 m}=-p}^{p} \bar{g}_{\nu, p}\left(x_{1}+j_{1} \Delta_{p}, \ldots, x_{2 m}+j_{2 m} \Delta_{p}\right) .
$$

Пусть $p$ - натуральное число, а $\varepsilon$ удовлетворяет неравенствам $0<\varepsilon<\Delta_{p} / 2$. Определим множество

$$
W_{p}(\varepsilon)=\bigcup_{j_{1}=-\infty}^{\infty} \cdots \bigcup_{j_{2 m}=-\infty}^{\infty} \prod_{k=1}^{2 m}\left[A_{p, 1}+\varepsilon+j_{k} \Delta_{p} ; A_{p, 2}-\varepsilon+j_{k} \Delta_{p}\right]
$$

ЛЕмма 6. Существует величина $C_{5}(p) \geqslant 1$ такая, что при $\nu>100$ для любой точки $\vec{x} \in \mathbb{T}^{2 m}$ иномеров $M_{i}$ таких, что выполняются неравенства

$$
M_{i}^{2} \leqslant 2 \nu, \quad i=1, \ldots, 2 m
$$

имеет место неравенство

$$
S_{\vec{M}}\left(h_{\nu, p}, \vec{x}\right)<C_{5}(p)\left(1+\ln \min _{i=1, \ldots, 2 m} M_{i}\right)^{m}
$$

ДОКАЗАТЕЛЬСТВО. В силу леммы 3 и определения функции $h_{\nu, p}$ можно взять $C_{5}(p)=$ $\max \left\{C_{2}^{m}(2 p+1)^{2 m}, 1\right\}$. Лемма доказана.

Положим $\varphi_{p}(t)=A_{p, 1}+\left(t\left(\bmod \Delta_{p}\right)\right)$. 
Лемма 7. Пусть $0<\varepsilon<\Delta_{p} / 2$. Тогда существует $\nu_{1}=\nu_{1}(\varepsilon, p)$ такое, что для любой точки $\vec{x} \in W_{p}(\varepsilon)$ выполнено

$$
\forall \nu>\nu_{1} \quad\left|S_{\vec{M}}\left(h_{\nu, p}, \vec{x}\right)\right| \geqslant \frac{3}{4} \ln ^{m} \nu
$$

для $M_{2 k-1}=\left[\nu \varphi_{p}\left(x_{2 k}\right)\right], M_{2 k}=\left[\nu \varphi_{p}\left(x_{2 k-1}\right)\right]$.

ДокАЗАТЕЛЬСТво. Из определения функции $h_{\nu, p}$ и лемм 4 и 5 следует, что при достаточно больших $\nu$ справедливо неравенство

$$
\left|S_{\vec{M}}\left(h_{\nu, p}, \vec{x}\right)\right| \geqslant \ln ^{m} \nu-\left((2 p+1)^{2} \max \left\{C_{3}(\varepsilon, p), C_{4}(\varepsilon, p)\right\}\right)^{m} \ln ^{m-1} \nu .
$$

Отсюда вытекает утверждение леммы.

3. Доказательство основной теоремы. Для дальнейшего нам понадобится следующее утверждение, принадлежащее Жижиашвили [9, с. 93].

ТЕОремА В. Если непрерывная функиия $n$ переменных $f(\vec{x})$ такова, что для некоторого $\varepsilon>0$ выполнены условия на частные модули непрерывности

$$
\omega_{i}\left(f, \delta_{i}\right)=\underset{\delta_{i} \rightarrow+0}{O}\left(\ln \left(\frac{1}{\delta_{i}}\right)^{-n-\varepsilon}\right), \quad i=1, \ldots, n,
$$

то ряд Фурье функиии $f$ равномерно сходится по Прингсхейму.

Пусть $p$ - натуральное число. Положим $\varepsilon_{p}=\Delta_{p} /(2(2 m+1))$. Обозначим через $V_{p}$ множество $W_{p}\left(\varepsilon_{p}\right)$, а через $V_{p, k}$ - его сдвиг на $2 \varepsilon_{p} k$ по каждой координате $(k=0, \ldots, 2 m)$.

Лемма 8. Для любого натурального р справедливо равенство

$$
\bigcup_{k=0}^{2 m} V_{p, k}=\mathbb{R}^{2 m}
$$

ДокАЗАТЕЛЬСТВо. В силу периодичности структуры множества $V_{p}$ достаточно доказать, что

$$
\bigcup_{k=0}^{2 m} V_{p, k} \supset\left[A_{p, 1}-\varepsilon_{p} ; A_{p, 2}-\varepsilon_{p}\right]^{2 m} .
$$

Рассмотрим попарно непересекающиеся интервалы

$$
\left(A_{p, 1}+(2 k-1) \varepsilon_{p}, \quad A_{p, 1}+(2 k+1) \varepsilon_{p}\right), \quad \text { где } k=0, \ldots, 2 m,
$$

лежащие в отрезке $\left[A_{p, 1}-\varepsilon_{p} ; A_{p, 2}-\varepsilon_{p}\right]$. Если $\vec{x} \in\left[A_{p, 1}-\varepsilon_{p} ; A_{p, 2}-\varepsilon_{p}\right]^{2 m}$, то среди $2 m+1$ указанных интервалов найдется хотя бы один $\left(k=k_{0}\right)$, не содержащий ни одного из $2 m$ чисел $x_{j}$. Тогда $\vec{x} \in V_{p, k_{0}}$. Лемма доказана.

В дальнейшем будем обозначать через $p_{q}$ и $r_{q}$ числа, определяемые представлением натурального числа $q \geqslant 2 m+2$ в виде

$$
q=(2 m+2) p_{q}+r_{q}, \quad \text { где } p_{q} \in \mathbb{N}, \quad r_{q} \in\{0, \ldots, 2 m+1\} .
$$

Перейдем непосредственно к доказательству теоремы. 
ДОКАЗАТЕЛЬСТВо ТЕОРЕМЫ 1 . Прежде всего, отметим, что для Любой точки $\vec{x}$ и для любых $\nu, p$ выполнено $\left|\bar{g}_{\nu, p}(\vec{x})\right| \leqslant 1$. Кроме того, для любой точки $\vec{x} \in \mathbb{T}^{2 m}$ существует не более $3^{2 m}$ слагаемьх в сумме (9), отличњых в этой точке от нуля. Поэтому для любой точки $\vec{x}$ и для любых $\nu, p$ выполнено $\left|h_{\nu, p}(\vec{x})\right| \leqslant 3^{2 m}$. Поскольку каждая функция $h_{\nu, p}(\vec{x})$ удовлетворяет условиям теоремы $\mathrm{B}$, то при достаточно больших (для данных $\nu$ и $p$ ) числах $M_{i}$ имеем

$$
\left|S_{\vec{M}}\left(h_{\nu, p}, \vec{x}\right)\right| \leqslant 3^{2 m}+1
$$

Построим теперь по индукции последовательность $\left\{N_{q}\right\}_{q=2 m+2}^{\infty}$.

Выберем $N_{2 m+2} \geqslant 100$ так, чтобы (здесь $\nu_{1}$ из леммы 7 )

$$
\begin{gathered}
\frac{3^{2 m}+1}{\ln ^{m} N_{2 m+2}}<\frac{1}{2^{2 m+2},} \\
N_{2 m+2}>\nu_{1}\left(\varepsilon_{1}, 1\right), \\
\frac{1}{N_{2 m+2}}<\Delta_{1}=A_{1,2}-A_{1,1} .
\end{gathered}
$$

Очередное $N_{q}$ выбираем столь большим, чтобы оно удовлетворяло следующим условиям:

$$
\begin{gathered}
N_{q}>\left(N_{q-1}\right)^{2}, \\
\frac{3^{2 m}+1}{\ln ^{m} N_{q}}<\frac{1}{2^{q}} \\
\frac{C_{5}\left(p_{q}\right)}{\ln ^{m} N_{q}}<\frac{1}{32 \ln ^{m} N_{q-1}}, \\
N_{q}>\nu_{1}\left(\varepsilon_{p_{q}}, p_{q}\right), \\
\forall \vec{M}: M_{i}>N_{q} \forall \vec{x}\left|S_{\vec{M}}\left(h_{N_{q-1}, p_{q-1}}, \vec{x}\right)\right| \leqslant 3^{2 m}+1, \\
\frac{1}{N_{q}}<\Delta_{p_{q}}=A_{p_{q}, 2}-A_{p_{q}}, 1 \\
\frac{N_{q}}{\ln ^{m} N_{q}} \geqslant 2 \frac{N_{q-1}}{\ln ^{m} N_{q-1}} .
\end{gathered}
$$

Отметим, что из $(15)$ следует (поскольку $C_{5}(p) \geqslant 1$ ) неравенство

$$
\frac{C_{5}\left(p_{q}\right)}{\ln ^{m} N_{q}}<\frac{C_{5}\left(p_{q-1}\right)}{4 \ln ^{m} N_{q-1}} .
$$

Положим теперь

$$
f_{q}(\vec{x})= \begin{cases}h_{N_{q}, p_{q}}\left(\vec{x}+r_{q} \varepsilon_{p_{q}} \vec{e}\right), & r_{q} \neq 2 m+1, \\ 0, & r_{q}=2 m+1,\end{cases}
$$

где $\vec{e}=(1, \ldots, 1) \in \mathbb{R}^{2 m}$.

Определим функцию

$$
F(\vec{x})=\sum_{q=2 m+2}^{\infty} \frac{f_{q}(\vec{x})}{\ln ^{m} N_{q}}
$$


Докажем, что для любого $\lambda>1$ ряд Фурье функции $F(\vec{x}) \lambda$-расходится всюду.

Зафиксируем $\lambda>1$. Будем считать $p_{*}$ столь большим, что

$$
\lambda_{p_{*}} \frac{N_{(2 m+2) p_{*}}}{N_{(2 m+2) p_{*}}-1}<\lambda .
$$

Отметим, что при $q \geqslant(2 m+2) p_{*}$ для любых $s$ и $t$ вьполнено

$$
\frac{1}{\lambda}<\frac{N_{q}-1}{N_{q} \lambda_{p_{q}}}<\frac{N_{q} \varphi_{p_{q}}(t)}{N_{q} \varphi_{p_{q}}(s)} \frac{N_{q}-1}{N_{q}} \leqslant \frac{\left[N_{q} \varphi_{p_{q}}(t)\right]}{\left[N_{q} \varphi_{p_{q}}(s)\right]} \leqslant \frac{N_{q} \varphi_{p_{q}}(t)}{N_{q} \varphi_{p_{q}}(s)} \frac{N_{q}}{N_{q}-1}<\frac{\lambda_{p_{q}} N_{q}}{N_{q}-1}<\lambda,
$$

т.е. любой набор номеров вида $\left\{\left[N_{q} \varphi_{p_{q}}\left(t_{k}\right)\right]\right\}_{k=1}^{2 m}$ входит в те, которые рассматриваются в определении $\lambda$-сходимости ряда.

Зафиксируем теперь точку $\vec{x} \in \mathbb{T}^{2 m}$. В силу леммы 8 для любого $p \geqslant p_{*}$ найдется такое $r^{*}=r^{*}(p, \vec{x}) \in\{0, \ldots, 2 m\}$, что $\vec{x} \in V_{p, r^{*}}$. Положим $q^{\prime}=q^{\prime}(p, \vec{x})=(2 m+2) p+r^{*}$ и $q^{\prime \prime}=q^{\prime \prime}(p)=(2 m+2) p+2 m+1$ (при этом $\left.p_{q^{\prime}}=p_{q^{\prime \prime}}=p\right)$.

Тогда по лемме 7 для $M_{2 k}=\left[N_{p} \varphi_{p}\left(x_{2 k-1}-r^{*} \varepsilon_{p}\right)\right], M_{2 k-1}=\left[N_{p} \varphi_{p}\left(x_{2 k}-r^{*} \varepsilon_{p}\right)\right] \mathrm{c}$ учетом (11) или (16) вьполнено

$$
\left|S_{\vec{M}}\left(f_{q^{\prime}}, \vec{x}\right)\right| \geqslant \frac{3}{4} \ln ^{m} N_{q^{\prime}}
$$

Поскольку ряд (21) сходится равномерно, то

$$
S_{\vec{M}}(F, \vec{x})=\sum_{q=2 m+2}^{\infty} \frac{S_{\vec{M}}\left(f_{q}, \vec{x}\right)}{\ln ^{m} N_{q}}
$$

Представим этот ряд в виде

$$
S_{\vec{M}}(F, \vec{x})=\sum_{q=2 m+2}^{q^{\prime}-1} \frac{S_{\vec{M}}\left(f_{q}, \vec{x}\right)}{\ln ^{m} N_{q}}+\frac{S_{\vec{M}}\left(f_{q^{\prime}}, \vec{x}\right)}{\ln ^{m} N_{q^{\prime}}}+\sum_{q=q^{\prime}+1}^{\infty} \frac{S_{\vec{M}}\left(f_{q}, \vec{x}\right)}{\ln ^{m} N_{q}}=S_{1}+S_{2}+S_{3} .
$$

В силу (22) имеем $\left|S_{2}\right| \geqslant 3 / 4$. Далее, в силу условий $(10),(14)$ и (17) вьполнено

$$
\left|S_{1}\right| \leqslant \sum_{q=2 m+2}^{q^{\prime}-1} \frac{3^{2 m}+1}{\ln ^{m} N_{q}} \leqslant \sum_{q=4}^{q^{\prime}-1} \frac{1}{2^{q}}<\frac{1}{8}
$$

Кроме того, в силу леммы 6 с учетом неравенства (20) вьполнено

$$
\left|S_{3}\right| \leqslant \sum_{q=q^{\prime}+1}^{\infty} \frac{C_{5}\left(p_{q}\right)\left(2+\ln N_{q^{\prime}}\right)^{m}}{\ln ^{m} N_{q}} \leqslant 2\left(2+\ln N_{q^{\prime}}\right)^{m} \frac{C_{5}\left(p_{q^{\prime}+1}\right)}{\ln ^{m} N_{q^{\prime}+1}}
$$

что в силу (15) дает

$$
\left|S_{3}\right| \leqslant \frac{2\left(2+\ln N_{q^{\prime}}\right)^{m}}{32 \ln ^{m} N_{q^{\prime}}}<\frac{1}{8}
$$


Окончательно получаем, что

$$
\left|S_{\vec{M}}(F, \vec{x})\right|>\frac{1}{2}
$$

С другой стороны, рассмотрим кубическую частичную сумму $S_{\vec{K}}(F, \vec{x})$ для $K_{j}=N_{q^{\prime \prime}}$, где по-прежнему $p \geqslant p_{*}$. Так как $f_{q^{\prime \prime}}(\vec{x}) \equiv 0$, то имеем

$$
S_{\vec{K}}(F, \vec{x})=\sum_{q=2 m+2}^{\infty} \frac{S_{\vec{K}}\left(f_{q}, \vec{x}\right)}{\ln ^{m} N_{q}}=\sum_{q=2 m+2}^{q^{\prime \prime}-1} \frac{S_{\vec{K}}\left(f_{q}, \vec{x}\right)}{\ln ^{m} N_{q}}+\sum_{q=q^{\prime \prime}+1}^{\infty} \frac{S_{\vec{K}}\left(f_{q}, \vec{x}\right)}{\ln ^{m} N_{q}}=S_{1}^{\prime}+S_{3}^{\prime} .
$$

По аналогии с предыдущим случаем получаем, что

$$
\left|S_{\vec{K}}(F, \vec{x})\right|<\frac{1}{4}
$$

Из оценок (23) и (24) при $p \rightarrow \infty$ следует $\lambda$-расходимость.

Докажем теперь оценку (1) на модуль непрерывности.

Рассмотрим приращение функции $F$; при этом достаточно рассмотреть случай, когда $F$ имеет приращение только по одной переменной, например по $x_{1}$. Итак, пусть $\delta>0$. Положим $\vec{y}=\left(x_{2}, \ldots, x_{2 m}\right)$. Имеем

$$
\Delta F=F\left(x_{1}+\delta, \vec{y}\right)-F\left(x_{1}, \vec{y}\right)=\sum_{q=2 m+2}^{\infty} \frac{f_{q}\left(x_{1}+\delta, \vec{y}\right)-f_{q}\left(x_{1}, \vec{y}\right)}{\ln ^{m} N_{q}}
$$

Предполагая $\delta$ достаточно малым $\left(\delta<1 / N_{2 m+2}\right)$, выберем натуральное $q_{0}$ так, чтобы

$$
\frac{1}{N_{q_{0}+1}} \leqslant \delta<\frac{1}{N_{q_{0}}}
$$

Имеем

$$
F\left(x_{1}+\delta, \vec{y}\right)-F\left(x_{1}, \vec{y}\right)=\left(\sum_{q=2 m+2}^{q_{0}}+\sum_{q=q_{0}+1}^{\infty}\right) \frac{f_{q}\left(x_{1}+\delta, \vec{y}\right)-f_{q}\left(x_{1}, \vec{y}\right)}{\ln ^{m} N_{q}}=S_{1}+S_{2} .
$$

Для второй суммы получаем с учетом условия (13):

$$
\left|S_{2}\right| \leqslant \sum_{q=q_{0}+1}^{\infty} \frac{\left|f_{q}\left(x_{1}+\delta, \vec{y}\right)-f_{q}\left(x_{1}, \vec{y}\right)\right|}{\ln ^{m} N_{q}} \leqslant \sum_{q=q_{0}+1}^{\infty} \frac{2 \cdot 3^{2 m}}{\ln ^{m} N_{q}} \leqslant \frac{4 \cdot 3^{2 m}}{\ln ^{m} N_{q_{0}+1}} \leqslant \frac{4 \cdot 3^{2 m}}{\ln ^{m}(1 / \delta)} .
$$

Перейдем к оценке первой суммы. Оценим приращение одной функции $f_{q}(\vec{x})$; при этом достаточно ограничиться случаем $r_{q}=0$, т.е. $f_{q}(\vec{x})=h_{N_{q}, p_{q}}(\vec{x})$, так как остальные $f_{q}$ отличаются только сдвигом аргумента. Отметим, что в силу условий (12) и (18) при $q \leqslant q_{0}$ вьполнено $\delta<1 / N_{q_{0}} \leqslant 1 / N_{q} \leqslant A_{p_{q}, 2}-A_{p_{q}, 1}$. Поэтому отрезок, соединяющий точки $\left(x_{1}, \vec{y}\right)$ и $\left(x_{1}+\delta, \vec{y}\right)$, пересекает не более двух сдвигов куба $\left(Q_{2,2, p_{q}}\right)^{m}$ на векторы $\left(j_{1} \Delta_{p_{q}}, \ldots, j_{2 m} \Delta_{p_{q}}\right)$, где $j_{k}=-p_{q}, \ldots, p_{q}$. Каждый такой куб пересекается с носителями ровно $3^{2 m}$ слагаемых в сумме $(9)$. Поэтому

$$
\left|f_{q}\left(x_{1}+\delta, \vec{y}\right)-f_{q}\left(x_{1}, \vec{y}\right)\right| \leqslant 2 \cdot 3^{2 m} X_{q}(\delta)
$$


где $X_{q}(\delta)$ есть верхняя грань приращений $\bar{g}_{N_{q}, p_{q}}(\vec{x})$ по переменной $x_{1}$ по отрезку длины не более $\delta$, целиком лежащему в одном из отрезков $\overline{I_{p_{q}, j}}$. Если $j=2$, то для некоторого $c \in\left[A_{p_{q}, 1}, A_{p_{q}, 2}\right]$ имеем

$$
\left|\bar{g}_{N_{q}, p_{q}}\left(x_{1}+\delta^{\prime}, \vec{y}\right)-\bar{g}_{N_{q}, p_{q}}\left(x_{1}, \vec{y}\right)\right| \leqslant\left|e^{i N_{q} \delta^{\prime} c}-1\right| \leqslant 2 \pi N_{q} \delta^{\prime} \leqslant 2 \pi N_{q} \delta
$$

так как длина хорды меньше длины дуги. Если же $j=1$ или $j=3$, то функция линейна по $x_{1}$ с угловьм коэффициентом $\alpha,|\alpha| \leqslant 1 / \Delta_{p_{q}}=1 /\left(A_{p_{q}, 2}-A_{p_{q}, 1}\right) \leqslant N_{q}$. Таким образом,

$$
X_{q}(\delta) \leqslant 2 \pi N_{q} \delta .
$$

Отметим, что функция $\left(\ln ^{m} t\right) / t$ монотонно убывает при достаточно больших $t$; поэтому при достаточно малых $\delta$ имеет место неравенство

$$
\delta \frac{N_{q_{0}}}{\ln ^{m} N_{q_{0}}}=\frac{\ln ^{m}(1 / \delta)}{1 / \delta} \frac{N_{q_{0}}}{\ln ^{m} N_{q_{0}}} \frac{1}{\ln ^{m}(1 / \delta)} \leqslant \frac{1}{\ln ^{m}(1 / \delta)} .
$$

Теперь с учетом (19) имеем

$$
\left|S_{1}\right| \leqslant 4 \pi \cdot 3^{2 m} \delta \sum_{q=2 m+2}^{q_{0}} \frac{N_{q}}{\ln ^{m} N_{q}} \leqslant 8 \pi \cdot 3^{2 m} \delta \frac{N_{q_{0}}}{\ln ^{m} N_{q_{0}}} \leqslant 8 \pi \cdot 3^{2 m} \frac{1}{\ln ^{m}(1 / \delta)} .
$$

Окончательно получаем

$$
|\Delta F| \leqslant(8 \pi+4) \cdot 3^{2 m} \frac{1}{\ln ^{m}(1 / \delta)}
$$

Оценка (1) доказана.

Автор выражает благодарность профессору М. И. Дьяченко за внимание к работе.

\section{СПИСОК ЦИТИРОВАННОЙ ЛИТЕРАТУРЫ}

[1] Fefferman C. On the convergence of multiple Fourier series // Bull. Amer. Math. Soc. 1971. V. 77. № 5. P. 744-745.

[2] Fefferman C. On the divergence of multiple Fourier series // Bull. Amer. Math. Soc. 1971. V. 77. № 2. P. 191-195.

[3] Бахбух М., Никишин Е. М. О сходимости двойных рядов Фурье от непрерьвных функций // Сиб. матем. ж. 1973. Т. 14. №6. С. 1189-1199.

[4] Осколков К.И. Оценка скорости приближения непрерьвной функции и ее сопряженной суммами Фурье на множестве полной меры // Изв. АН СССР. Сер. матем. 1974. Т. 38. №6. C. $1373-1407$.

[5] ЖКижиашвили Л. В. О сходимости кратных тригонометрических рядов Фурье // Сообщ. АН ГССР. 1975. Т. 80. №1. С. 17-19.

[6] Дьяченко М. И. Некоторые проблемы теории кратных тригонометрических рядов // УМН. 1992. Т. 47. № 5. С. $97-162$.

[7] Бахвалов А. Н. О расходимости всюду рядов Фурье непрерывных функций многих переменных // Матем. сб. 1997. Т. 188. № 8. С. 45-62.

[8] Bojanic R. An estimate of the rate of convergence for Fourier series of functions of bounded variation // Publ. Inst. Math. (Belgrade). 1979. V. 26. № 40. P. 57-60.

[9] ЖКижиашвили Л. В. О некоторых вопросах из теории простых и кратных тригонометрических рядов // УМН. 1973. Т. 28. № 2. С. 65-119. 